\title{
Normalized Bicoprime Factorizations
}

DOI:

10.23919/ECC.2018.8550529

\section{Document Version}

Accepted author manuscript

Link to publication record in Manchester Research Explorer

\section{Citation for published version (APA):}

Tsiakkas, M., \& Lanzon, A. (2018). Normalized Bicoprime Factorizations. In Proceedings of the 17th European Control Conference, Limassol, Cyprus, June 2018 https://doi.org/10.23919/ECC.2018.8550529

\section{Published in:}

Proceedings of the 17th European Control Conference, Limassol, Cyprus, June 2018

\section{Citing this paper}

Please note that where the full-text provided on Manchester Research Explorer is the Author Accepted Manuscript or Proof version this may differ from the final Published version. If citing, it is advised that you check and use the publisher's definitive version.

\section{General rights}

Copyright and moral rights for the publications made accessible in the Research Explorer are retained by the authors and/or other copyright owners and it is a condition of accessing publications that users recognise and abide by the legal requirements associated with these rights.

\section{Takedown policy}

If you believe that this document breaches copyright please refer to the University of Manchester's Takedown Procedures [http://man.ac.uk/04Y6Bo] or contact uml.scholarlycommunications@manchester.ac.uk providing relevant details, so we can investigate your claim.

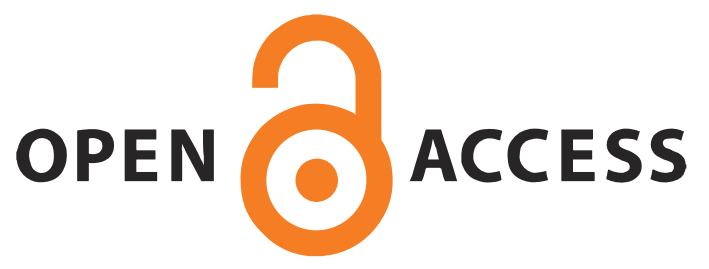




\title{
Normalized Bicoprime Factorizations
}

\author{
Mihalis Tsiakkas ${ }^{1}$ and Alexander Lanzon ${ }^{2}$
}

\begin{abstract}
This paper introduces the concept of normalized bicoprime factorizations. The notion of a normalized bicoprime factorization is first defined and conditions for normalization are given in state space. Algorithms that can be used to obtain a normalized bicoprime factorization of the plant are presented.
\end{abstract}

\section{INTRODUCTION}

Normalized coprime factorizations play an important role in control theory. It is well known that introducing the normalization property to a coprime factorization of a plant offers many advantages, with a prominent one being the ability to directly derive a lower bound on the achievable robust stability margin [1], [2]. This then allows for the construction of a robustly stabilizing controller without the need of an iterative procedure. The well known $\mathscr{H}_{\infty}$ loopshaping design procedure uses normalized coprime factorizations [3] to robustly stabilize the shaped plant. Another result making use of normalized coprime factorizations is the $\nu$-gap metric [4], [5], which is a powerful tool in robustness analysis.

A right (resp. left) coprime factorization of a plant is said to be normalized if the associated graph symbol is inner (resp. co-inner) [2]. Equivalently, let the pair $\{N, M\}$ be a right coprime factorization of $P=N M^{-1} \in \mathscr{R}$ over $\mathscr{R} \mathscr{H}_{\infty}$, then the factorization is normalized if $M^{\sim} M+N^{\sim} N=I$, where $T^{\sim}$ denotes the $\mathscr{L}_{2}$-adjoint of $T$ defined as $T^{\sim}=$ $T^{T}(-s)$ [6, Definition 3.8].

Bicoprime factorizations (BCFs) of the plant were first introduced by Vidyasagar in [7]. They are a generalization on the aforementioned coprime factorizations. BCFs were briefly studied in the late 80's however they were quickly abandoned due to high mathematical complexity when their coprime factor counterparts began to yield powerful results. Recent work [8], [9] has shown that it is possible to capture and generalize earlier coprime factor results into a more complete BCF framework. Several BCF results were presented therein mostly pertaining to internal stability and $\mathrm{BCF}$ parameterization.

Just like the classical case of left and right coprime factorizations, every plant in $\mathscr{R}$ admits a BCF over $\mathscr{R} \mathscr{H}_{\infty}$. The ordered quad $\{N, M, L, K\}$ is said to be bicoprime (BC) in $\mathscr{R} \mathscr{H}_{\infty}$ if $\{L, M\}$ is left coprime (LC) in $\mathscr{R} \mathscr{H}_{\infty},\{N, M\}$ is right coprime (RC) in $\mathscr{R} \mathscr{H}_{\infty}$ and $K \in \mathscr{R} \mathscr{H}_{\infty}$. Furthermore,

\footnotetext{
${ }^{1}$ Mihalis Tsiakkas is with the KIOS Research and Innovation Center of Excellence, University of Cyprus, Nicosia, Cyprus m.tsiakkas@ieee.org

${ }^{2}$ Alexander Lanzon is with the Control Systems Centre, School of Electrical and Electronic Engineering, University of Manchester, Manchester, M13 9PL, UK a.lanzon@ieee.org

This work was supported by the Engineering and Physical Sciences Research Council (EPSRC) [grant number EP/R008876/1]. All research data supporting this publication are directly available within this publication.
}

it is a $\mathrm{BCF}$ of $P \in \mathscr{R}$ over $\mathscr{R} \mathscr{H}_{\infty}$ if $M$ is square and invertible in $\mathscr{R}$ and $P=N M^{-1} L+K$. Such a structure is commonly encountered in many areas of control theory, including standard state space realizations and basic closed loop transfer functions of feedback interconnections. It can be shown in fact that these are BCFs over sets other than $\mathscr{R} \mathscr{H}_{\infty}$, for example a minimal state space realization of a plant is a $\mathrm{BCF}$ over the ring of polynomials $\mathbb{R}[s]$ [7].

BCFs have been shown to provide benefits in several areas of interest to the control community. For example, in internal stability analysis, BCFs can induce tests based on the invertibility of objects with dimensions smaller that those of the plant [9]; comparatively, coprime factor tests require the inversion of transfer matrices with dimensions equal to the number of inputs or outputs of the plant [6, Lemma 5.2]. It can also be shown that BCFs can be successfully utilized in the context of robust control synthesis with one significant benefit being a reduction in the order of the Algebraic Riccati Equations (AREs) that need to be solved for the construction of a robustly stabilizing controller. Even though this result is interesting in its own right, it is outside the scope of this paper and will be published elsewhere.

This paper develops the idea of normalized BCFs in a manner similar to classical coprime factorizations with the left and right coprime pairs of the factorization both being normalized. Subsequently, methods of obtaining a normalized BCF of a plant are outlined. Finally, a numerical example is provided to illustrate the applicability of the theory developed herein.

\section{PRELIMINARIES}

In this section some preliminary results and notation are presented. These will be used as a basis for the main results of this paper developed in the subsequent sections.

Definition 1: The set of all $\mathrm{BC}$ quads in $\mathscr{R} \mathscr{H}_{\infty}$ is denoted by $\mathscr{B}$. The set of all BCFs of a plant $P \in \mathscr{R}$ over $\mathscr{R} \mathscr{H}_{\infty}$ is denoted by $\mathscr{B}(P)$.

It is often convenient to pack a $\mathrm{BC}$ quad into a matrix as in the following definition.

Definition 2: The set $\tilde{\mathscr{B}}$ is defined as

$$
\tilde{\mathscr{B}}=\left\{\left[\begin{array}{cc}
M & -L \\
N & K
\end{array}\right]:\{N, M, L, K\} \in \tilde{\mathscr{B}}\right\} .
$$

The set of all objects in $\tilde{\mathscr{B}}$ that define a BCF of a plant $P \in \mathscr{R}$ is denoted by $\tilde{\mathscr{B}}(P)$. Objects in $\tilde{\mathscr{B}}(P)$ are referred to as BCF symbols of $P$.

It should be noted that BCF symbols are not equivalent to graph symbols as induced by coprime factorizations as they cannot be used to generate all bounded input-output pairs. 
They do however share some properties such as common transmission zero set with the plant as shown by [9].

An appealing feature of coprime factorizations is that they can easily be obtained from state space data of a plant using the formulae given by [10], which were extended to the normalized case by [11] and [12]. The following lemma gives a state space characterization of BCFs for a plant.

Lemma 1 ([9]): Let $P \in \mathscr{R}^{p \times q}$ have a stabilizable and detectable state space realization $P=\left[\begin{array}{l|l}A & B \\ \hline C & D\end{array}\right]$ where $A \in \mathbb{R}^{n \times n}$. Furthermore, suppose that $Q \in \mathbb{R}^{n \times r}$ and $R \in$ $\mathbb{R}^{r \times n}$ are such that $A+Q R$ is Hurwitz and let $D_{N} \in \mathbb{R}^{p \times r}$ and $D_{L} \in \mathbb{R}^{r \times q}$ be arbitrarily chosen matrices. Then

$$
\left[\begin{array}{c|c:c}
A+Q R & Q & B+Q D_{L} \\
\hline R & I & D_{L} \\
\hdashline C+D_{N} R & D_{N} & D+D_{N} D_{L}
\end{array}\right] \in \tilde{\mathscr{B}}(P) \text {. }
$$

It is apparent that the above BCF parametrization could be simplified by selecting $D_{N}=0$ and $D_{L}=0$. This restriction will be imposed for the remainder of this paper. The results presented in the subsequent sections can be derived for the case of non-zero $D_{N}$ and $D_{L}$ via some simple, albeit tedious, linear algebra. When the above restriction is imposed, (1) will be referred to as a $Q R$-BCF parametrization of $P$.

The controllability and observability Gramians of a plant $P \in \mathscr{R} \mathscr{H}_{\infty}$, with a stabilizable and detectable state space realization $P=\left[\begin{array}{l|l}A & B \\ \hline C & D\end{array}\right]$, are given by the solutions $X \geq$ 0 and $Y \geq 0$ to the Lyapunov equations

$$
\begin{gathered}
A X+X A^{*}+B B^{*}=0 \text { and } \\
Y A+A^{*} Y+C^{*} C=0
\end{gathered}
$$

respectively [6]. These concepts will be used in the definition of normalized BCFs.

\section{NORMALIZED BCF}

In this section the notion of a normalized BCF of a plant is formally defined. Some state space conditions are provided that help to establish whether a BCF is normalized. These are then used as a basis for algorithms that attempt to generate such a BCF for a given plant in the next section.

Definition 3: Let $P \in \mathscr{R}^{p \times q}$ have a BCF $\{N, M, L, K\} \in$ $\mathscr{B}(P)$. The $\mathrm{BCF}$ is said to be normalized if the factors satisfy

$$
\begin{gathered}
M^{\sim} M+N^{\sim} N=I, \\
M M^{\sim}+L L^{\sim}=I .
\end{gathered}
$$

Though the above definition of a normalized BCF involves normalized coprime pairs, it is important to realize that this new concept is distinct from the classically studied normalized coprime factorizations.

Before presenting state space conditions for the normalization of a $Q R$-BCF, the following terminology is needed. The triplet $(C, A, B)$ is said to have no modes that are both controllable and observable when all controllable modes in $(A, B)$ are unobservable in $(C, A)$ and all observable modes in $(C, A)$ are uncontrollable in $(A, B)$. On the other hand,
$(C, A, B)$ is said to be minimal if $(A, B)$ is controllable and $(C, A)$ is observable.

The following theorem gives necessary and sufficient state space conditions for a $Q R$-BCF to be normalized.

Theorem 1: Let $P \in \mathscr{R}^{p \times q}$ have a stabilizable and detectable state space realization $P=\left[\begin{array}{c|c}A & B \\ \hline C & D\end{array}\right]$ where $A \in \mathbb{R}^{n \times n}$ and let $Q \in \mathbb{R}^{n \times r}$ and $R \in \mathbb{R}^{r \times n}$ be such that $A+Q R$ is Hurwitz. Define

$$
\begin{aligned}
G & =\left[\begin{array}{c:c}
M & -L \\
\hdashline N & K
\end{array}\right] \\
& =\left[\begin{array}{c|c:c}
A+Q R & Q & B \\
\hline R & I & 0 \\
\hdashline C^{-} & 0 & D
\end{array}\right] \in \tilde{\mathscr{B}}(P) .
\end{aligned}
$$

Then the $Q R$-BCF defined by $G$ is normalized if and only if each of the triplets $\left(R, A+Q R, Q+X R^{*}\right)$ and $(R+$ $\left.Q^{*} Y, A+Q R, Q\right)$ has no modes that are both controllable and observable, where $X \geq 0$ and $Y \geq 0$ are the controllability and observability Gramians of $G$ respectively.

Proof: By direct calculation it can be shown that

$$
\left[\begin{array}{c}
M \\
N
\end{array}\right]^{\sim}\left[\begin{array}{c}
M \\
N
\end{array}\right]=I+\left[\begin{array}{c|c}
A+Q R & Q \\
\hline R+Q^{*} Y & 0
\end{array}\right]+\left[\begin{array}{c|c}
A+Q R & Q \\
\hline R+Q^{*} Y & 0
\end{array}\right]^{\sim}
$$

Given that for any strictly proper $S \in \mathscr{R} \mathscr{H}_{\infty}, S+S^{\sim}=0$ if and only if $S=0$, it follows that (5) reduces to the identity matrix if and only if $\left[\begin{array}{c|c}A+Q R & Q \\ \hline R+Q^{*} Y & 0\end{array}\right]=0$ or equivalently, using [6, Theorem 3.10], the triplet $\left(R+Q^{*} Y, A+Q R, Q\right)$ has no modes that are both controllable and observable. It can similarly be proven that $\{L, M\}$ is normalized if and only if $\left(R, A+Q R, Q+X R^{*}\right)$ has no modes that are both controllable and observable.

Corollary 1: Suppose that $Q$ and $R$ in Theorem 1 are chosen such that $(R, A, Q)$ is minimal. Then the $Q R$-BCF induced by $G$ in (4) is normalized if and only if $Q+X R^{*}=0$ and $R+Q^{*} Y=0$.

Proof: First note that

$$
\left[\begin{array}{c}
M \\
N
\end{array}\right]=\left[\begin{array}{c|c}
A+Q R & Q \\
\hline R & I \\
C & 0
\end{array}\right] \in \mathscr{R} \mathscr{H}_{\infty}
$$

is minimal and that its observability Gramian is given by $Y$.

Then by definition, the $\mathrm{RC}$ pair $\{N, M\}$ is normalized if and only if

$$
\begin{aligned}
{\left[\begin{array}{c}
M \\
N
\end{array}\right]^{\sim}\left[\begin{array}{c}
M \\
N
\end{array}\right]=I } & \Longleftrightarrow\left[\begin{array}{l}
M \\
N
\end{array}\right] \text { is inner } \\
& \Longleftrightarrow R+Q^{*} Y=0
\end{aligned}
$$

where the last equivalence follows from [6, Corollary 13.30]. The fact that the LC pair $\{M, L\}$ is normalized if and only if $Q+X R^{*}=0$ can be similarly proven.

Remark 1: It is easy to see that $Q+X R^{*}=0$ and $R+Q^{*} Y=0$ are sufficient for the induced $Q R$-BCF to be normalized regardless of the minimality of $(R, A, Q)$. 
The following theorem gives a sufficient condition for a $Q R$-BCF to be normalized based on two AREs with signdefinite quadratic terms. The resulting condition, although not necessary, is easier to evaluate than that of Theorem 1, making it more useful in constructing normalized BCFs.

Theorem 2: Let $P \in \mathscr{R}^{p \times q}$ have a stabilizable and detectable state space realization $P=\left[\begin{array}{c|c}A & B \\ \hline C & D\end{array}\right]$ where $A \in \mathbb{R}^{n \times n}$. Let $Q \in \mathbb{R}^{n \times r}$ and $R \in \mathbb{R}^{r \times n}$ be such that $(A, Q)$ is stabilizable and $(R, A)$ is detectable. Furthermore, let $X \geq 0$ and $Y \geq 0$ be the stabilizing solutions to

$$
\begin{gathered}
X A^{*}+A X-X R^{*} R X+B B^{*}=0 \text { and } \\
Y A+A^{*} Y-Y Q Q^{*} Y+C^{*} C=0 .
\end{gathered}
$$

Finally, suppose that $Q+X R^{*}=0$ and $R+Q^{*} Y=0$. Then the $Q R$-BCF induced by $Q$ and $R$ is normalized.

Proof: First note that $A+Q R$ is Hurwitz, therefore $Q$ and $R$ induce a valid $Q R$-BCF. Then by substituting $Q=$ $-X R^{*}$ and $R=-Q^{*} Y$ into (6) and (7) respectively, it can be seen that $X$ and $Y$ are the controllability and observability Gramians of the associated BCF symbol respectively. The conclusion then follows from Theorem 1.

The following lemma relates the normalizing $Q$ and $R$ to the Gramians of the associated BCF symbol. The result proves useful in defining terminating criteria for the iterative algorithms used to obtain a normalized $Q R$-BCF.

Lemma 2: Let $P \in \mathscr{R}^{p \times q}$ have a stabilizable and detectable state space realization $P=\left[\begin{array}{l|l}A & B \\ \hline C & D\end{array}\right]$ and a normalized $Q R$-BCF induced by the matrices $Q$ and $R$ satisfying the conditions of Theorem 2. Then

$$
\left[\begin{array}{c}
Q \\
R^{*}
\end{array}\right] \in \operatorname{Ker}\left[\begin{array}{cc}
I & X \\
Y & I
\end{array}\right]
$$

Furthermore, $Q \in \operatorname{Ker}(I-X Y)$ and $R^{*} \in \operatorname{Ker}(I-Y X)$.

Proof: The proof follows trivially from $Q+X R^{*}=0$ and $R+Q^{*} Y=0$.

As a result of the above lemma, it follows that for any normalized $Q R$-BCF of the type being considered

$$
\|(I-X Y) Q\|=0 \text { and }\|R(I-X Y)\|=0 .
$$

\section{Constructing a Normalized BCF}

Obtaining a normalized left or right coprime factorization of a plant is simple and requires the solution of a single ARE with a sign-definite quadratic term as demonstrated by [11], [12]. On the contrary, BCFs require the solution of two coupled AREs, which is a considerably harder problem, a direct solution to which does not exist in general. Though the normalization condition set forth by Theorem 2 appears to be simple, it must be noted that it is in fact hard to solve as the coupling between the two AREs leads to a nonlinear problem. This can be observed by substituting for and eliminating $Q$ and $R$ from (6) and (7) yielding

$$
\begin{gathered}
X A_{R}^{*}+A_{R} X-X C^{*} C X+B B^{*}=0, \\
A_{Q}^{*} Y+Y A_{Q}-Y B B^{*} Y+C^{*} C=0
\end{gathered}
$$

where $A_{R}=(I-X Y) A$ and $A_{Q}=A(I-X Y)$, which must be solved for $X \geq 0$ and $Y \geq 0$. No explicit mathematical method exists in the literature to solve such coupled AREs.

In this section, two iterative algorithms are presented that attempt to generate a pair of matrices $Q$ and $R$ satisfying the conditions set forth in Theorem 2.

Algorithm 1: Let $P \in \mathscr{R}^{p \times q}$ have a stabilizable and detectable state space realization $P=\left[\begin{array}{c|c}A & B \\ \hline C & D\end{array}\right]$ where $A \in \mathbb{R}^{n \times n}$ and specify a tolerance $\mu>0$.

1) Select $R_{0} \in \mathbb{R}^{r \times n}$ such that $\left(R_{0}, A\right)$ is detectable and set $i=0$.

2) Solve the ARE

$$
X_{i} A^{*}+A X_{i}-X_{i} R_{i}^{*} R_{i} X_{i}+B B^{*}=0
$$

for the stabilizing solution $X_{i} \geq 0$ and set $Q_{i+1}=$ $-X_{i} R_{i}^{*}$.

3) Solve the ARE

$$
Y_{i} A+A^{*} Y_{i}-Y_{i} Q_{i+1} Q_{i+1}^{*} Y_{i}+C^{*} C=0
$$

for the stabilizing solution $Y_{i} \geq 0$ and set $R_{i+1}=$ $-Q_{i+1}^{*} Y_{i}$.

4) If $\max \left\{\left\|R_{i}\left(I-X_{i} Y_{i}\right)\right\|,\left\|\left(I-X_{i} Y_{i-1}\right) Q_{i}\right\|\right\}<\mu$ and $i \geq 1$, stop. Otherwise increment $i$ and go to (2).

The following algorithm is inspired by the Kleinman approach to solving AREs [13], where the solution is obtained via the recursive solution of Lyapunov equations. For brevity, the notation $A_{\{i, j\}}=A+Q_{i} R_{j}$ will be used.

Algorithm 2: Let $P \in \mathscr{R}^{p \times q}$ have a stabilizable and detectable state space realization $P=\left[\begin{array}{l|l}A & B \\ \hline C & D\end{array}\right]$ where $A \in \mathbb{R}^{n \times n}$ and specify a tolerance $\mu>0$.

1) Select $Q_{0} \in \mathbb{R}^{n \times r}$ and $R_{0} \in \mathbb{R}^{r \times n}$ such that $A+Q_{0} R_{0}$ is Hurwitz and set $i=0$.

2) Solve the Lyapunov equation

$$
X_{i} A_{\{i, i\}}^{*}+A_{\{i, i\}} X_{i}+Q_{i} Q_{i}^{*}+B B^{*}=0
$$

for the solution $X_{i} \geq 0$ and set $Q_{i+1}=-X_{i} R_{i}^{*}$.

3) Solve the Lyapunov equation

$$
Y_{i} A_{\{i+1, i\}}+A_{\{i+1, i\}}^{*} Y_{i}+R_{i}^{*} R_{i}+C^{*} C=0
$$

for the solution $Y_{i} \geq 0$ and set $R_{i+1}=-Q_{i+1}^{*} Y_{i}$.

4) If $\max \left\{\left\|R_{i}\left(I-X_{i} Y_{i}\right)\right\|,\left\|\left(I-X_{i} Y_{i-1}\right) Q_{i}\right\|\right\}<\mu$ and $i \geq 1$, stop. Otherwise increment $i$ and go to (2).

The suitability of the stopping conditions of Algorithms 1 and 2 could be explained using Lemma 2. Another more intuitive approach is as follows. Consider Algorithm 1, then

$$
\begin{aligned}
R_{i+1}-R_{i} & =-Q_{i+1}^{*} Y_{i}-R_{i} \\
& =R_{i} X_{i} Y_{i}-R_{i} \\
& =-R_{i}\left(I-X_{i} Y_{i}\right) .
\end{aligned}
$$

It can similarly be shown that

$$
Q_{i+1}-Q_{i}=-\left(I-X_{i} Y_{i-1}\right) Q_{i} .
$$

Hence, taking the norm of (13) and (14) gives a measure of the change imparted on $R_{i}$ and $Q_{i}$ at the $i^{t h}$ iteration 
respectively. Therefore as (13) and (14) tend to $0, Q_{i}$ and $R_{i}$ converge to constant values. It can be shown that the same arguments hold for Algorithm 2 for all $i>0$.

A seemingly reasonable change to Algorithm 2 would be to replace (12) with

$$
Y_{i} A_{\{i, i\}}+A_{\{i, i\}}^{*} Y_{i}+R_{i}^{*} R_{i}+C^{*} C=0
$$

and then update $R_{i+1}=-Q_{i}^{*} Y_{i}$. However by doing this (13) and (14) no longer hold. This means that $Q_{i+1}$ and $R_{i+1}$ are not related to their previous values in a simple way. Though extensive numerical testing indicates that the resulting algorithm also converges to the normalizing $Q$ and $R$, it generally takes longer to converge than the proposed two algorithms and is not as numerically reliable.

An important point to make about the above two algorithms is that a solution always exists at every iteration. For Algorithm 1 this is immediately evident by noting that $\left(A, Q_{i}\right)$ is stabilizable for all $i \geq 1$ and $\left(R_{i+1}, A\right)$ is respectively detectable for all $i \geq 0$ and then using [6, Theorem 13.7]. The same fact can be proven for Algorithm 2, but the following lemma is first needed.

Lemma 3: Let $P \in \mathscr{R}^{p \times q}$ have a stabilizable state space realization $P=\left[\begin{array}{c|c}A & B \\ \hline C & D\end{array}\right]$ where $A \in \mathbb{R}^{n \times n}$ and suppose that $F \in \mathbb{R}^{q \times n}$ is such that $A+B F$ is Hurwitz. Furthermore, let $Y \geq 0$ be the solution to the Lyapunov equation

$$
(A+B F)^{*} Y+Y(A+B F)+F^{*} F+C^{*} C=0 .
$$

Then $A-B B^{*} Y$ is also Hurwitz.

Proof: First define

$$
S=\frac{1}{\sqrt{2}}\left[\begin{array}{c}
2 B^{*} Y+F \\
\sqrt{2} C \\
F
\end{array}\right]
$$

and note that $\left(S, A-B B^{*} Y\right)$ is detectable. Then by rearranging (15) it can be shown that $Y$ is also a solution to

$$
\left(A-B B^{*} Y\right)^{*} Y+Y\left(A-B B^{*} Y\right)+S^{*} S=0
$$

The conclusion then follows from [14, Theorem 13.24].

Lemma 4: Let $P \in \mathscr{R}^{p \times q}$ have a stabilizable and detectable state space realization $P=\left[\begin{array}{l|l}A & B \\ \hline C & D\end{array}\right]$. Now consider Algorithm 2 applied to this state space realization of $P$. Then there exists a $X_{i} \geq 0$ and $Y_{i} \geq 0$ satisfying (11) and (12) respectively for all $i \geq 0$.

Proof: First note that $R_{i}^{*} R_{i}+C^{*} C \geq 0$ and $Q_{i} Q_{i}^{*}+$ $B B^{*} \geq 0$ for all $i \geq 0$. Then using Lemma 3 it follows that $A_{\{i, i\}}$ and $A_{\{i, i+1\}}$ are Hurwitz for all $i \geq 0$. This then guarantees via [14, Theorem 13.21] that there exist $X_{i} \geq 0$ and $Y_{i} \geq 0$ satisfying (11) and (12) or all $i \geq 0$, which concludes the proof.

Algorithms used to iteratively solve single AREs typically exhibit monotonicity in the iteration variables [13], [15], [16], a fact exploited in proving that they do converge to the stabilizing solution (if it exists). Unfortunately, this is not always the case for the proposed algorithms, thus a simple proof of convergence is not available. This can be seen by very simple counter examples such as the $1^{\text {st }}$ order plant

$$
P=\left[\begin{array}{l|l}
1 & 1 \\
\hline 1 & 0
\end{array}\right]
$$

with $Q_{0}=3$ and $R_{0}=-2$. Figure 1 demonstrates this nonmonotonic behavior when using either algorithm to obtain a normalized $\mathrm{BCF}$ of the above plant with the given initial conditions and tolerance $\mu=10^{-3}$.

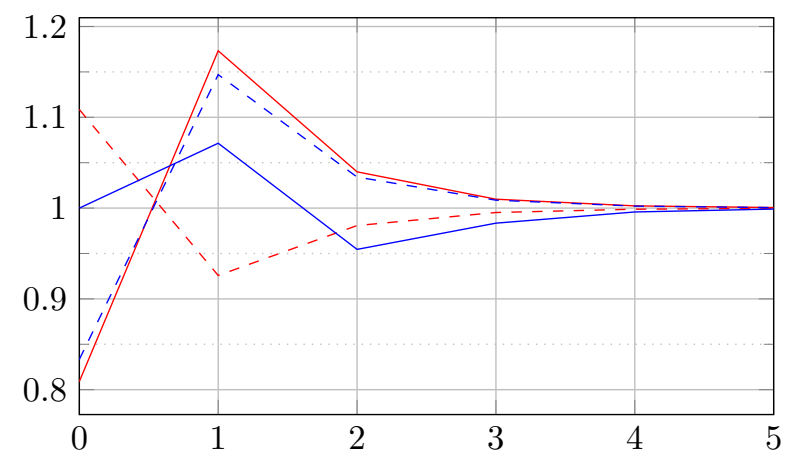

Fig. 1. Non-monotonic behavior in Algorithm 1 ( - ) and Algorithm $2(-)$. Solid lines correspond to $\rho\left(X_{i} X_{i-1}^{-1}\right)$ and dashed lines to $\rho\left(Y_{i} Y_{i-1}^{-1}\right)$.

Even though no convergence proof is provided for either algorithm, no cases were found where they fail to converge to a normalizing solution.

The selection of initial conditions for the above algorithms is important. Suppose that for a plant $P \in \mathscr{R}^{p \times q}, Q$ and $R$ induce a normalized $Q R$-BCF. If $R_{0}$ is chosen to be exactly $R$, then Algorithm 1 would converge after just one iteration. On the other hand, Algorithm 2 would need a few iterations depending on how close $Q_{0}$ is to the actual solution $Q$. It is also possible that $R_{i}$ is changed before re-converging to the solution. If also $Q_{0}$ is chosen as $Q$, then Algorithm 2 would also converge after just one iteration.

The following lemma gives a necessary condition $Q$ and $R$ must satisfy in order for a BCF to be normalized.

Lemma 5: Let $P \in \mathscr{R}^{p \times q}$ have a stabilizable and detectable state space realization $P=\left[\begin{array}{c|c}A & B \\ \hline C & D\end{array}\right]$ and a normalized $Q R$-BCF given by Theorem 2 . Then there exists a unitary matrix $U$ such that

$$
C Q=(R B U)^{*} \text {. }
$$

Proof: Note that (6) can be rearranged into $Q Q^{*}=$ $X A^{*}+A X+B B^{*}$. Substituting into (7) yields

$$
\begin{gathered}
Y\left(X A^{*}+A X+B B^{*}\right) Y=A^{*} Y+Y A+C^{*} C \\
Y B B^{*} Y=(I-Y X) A^{*} Y+Y A(I-X Y)+C^{*} C .
\end{gathered}
$$

Then pre- and post-multiplying by $Q^{*}$ and $Q$ respectively and using Lemma 2, gives $Q^{*} Y B B^{*} Y Q=Q^{*} C^{*} C Q$, or equivalently $R B B^{*} R^{*}=Q^{*} C^{*} C Q$. The conclusion then follows from [17, Theorem 7.3.11]. 


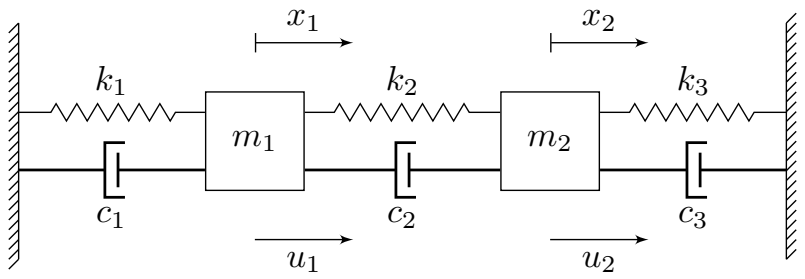

Fig. 2. Mass-spring-damper system schematic.

\section{NUMERICAL EXAMPLE}

For this example we consider a mass-spring-damper system extensively studied as a benchmark in many areas of control theory (see for example [16] or [5]).

A schematic representation of the system under consideration is shown in Figure 2. Two masses are attached to the walls and coupled together via springs and dampers. It is assumed that the masses are constrained to slide only in the horizontal direction while the surface upon which they do so is frictionless. The inputs to the system are the forces $u_{1}$ and $u_{2}$ applied to $m_{1}$ and $m_{2}$ respectively while the outputs are their respective displacements $x_{1}$ and $x_{2}$.

The parameters used were $k_{1}=0.5 \mathrm{~N} / \mathrm{m}, k_{2}=1.75 \mathrm{~N} / \mathrm{m}$, $k_{3}=1.5 \mathrm{~N} / \mathrm{m}, c_{1}=0.1 \mathrm{Ns} / \mathrm{m}, c_{2}=0.3 \mathrm{Ns} / \mathrm{m}, c_{3}=0.5$ Ns $/ \mathrm{m}, m_{1}=1.25 \mathrm{~kg}$ and $m_{2}=1 \mathrm{~kg}$. With the above values, a state space realization of the plant can be obtained as

$$
P=\left[\begin{array}{cccc|cc}
0 & 0 & 1 & 0 & 0 & 0 \\
0 & 0 & 0 & 1 & 0 & 0 \\
-1.8 & 1.4 & -0.32 & 0.24 & 0.8 & 0 \\
1.75 & -3.25 & 0.3 & -0.8 & 0 & 1 \\
\hline 1 & 0 & 0 & 0 & 0 & 0 \\
0 & 1 & 0 & 0 & 0 & 0
\end{array}\right] .
$$

Algorithms 1 and 2 were executed for the above state space realization of $P$ with the tolerance set to $\mu=10^{-3}$ and using as initial conditions

$$
Q_{0}=\left[\begin{array}{llll}
0 & 0 & 0.5 & 0.5
\end{array}\right]^{*} \text { and } R_{0}=-\left[\begin{array}{llll}
0.3 & 0.2 & 0 & 0
\end{array}\right] \text {. }
$$

It can be easily shown that $Q_{0}$ and $R_{0}$ satisfy the necessary condition given by Lemma 5 , with $Q_{0}^{*} C^{*} C Q_{0}=$ $R_{0} B B^{*} R_{0}^{*}=0$.

Table I provides some important data obtained from the execution of the two algorithms including the number of iterations it took for each to converge to its final state. The trajectories of $\left\|R_{i}\left(I-X_{i} Y_{i}\right)\right\|$ and $\left\|\left(I-X_{i} Y_{i-1}\right) Q_{i}\right\|$ are shown in Figure 3 and Figure 4 for both algorithms. The results are plotted on linear and logarithmic scales

\begin{tabular}{ccc}
\hline Parameter & Algorithm 1 & Algorithm 2 \\
\hline Iterations & 7 & 7 \\
Time & $11.3 \mathrm{~ms}$ & $3.1 \mathrm{~ms}$ \\
$\left\|R_{i}\left(I-X_{i} Y_{i}\right)\right\|$ & $2.421 \times 10^{-4}$ & $2.779 \times 10^{-4}$ \\
$\left\|\left(I-X_{i} Y_{i-1}\right) Q_{i}\right\|$ & $4.069 \times 10^{-4}$ & $4.659 \times 10^{-4}$ \\
$\{L, M\}$ normalization error & $1.7246 \times 10^{-4}$ & $1.9754 \times 10^{-4}$ \\
$\{N, M\}$ normalization error & $8.4916 \times 10^{-16}$ & $5.8412 \times 10^{-8}$ \\
\hline
\end{tabular}

TABLE I

EXECUTION DATA FOR Algorithms 1 AND 2.

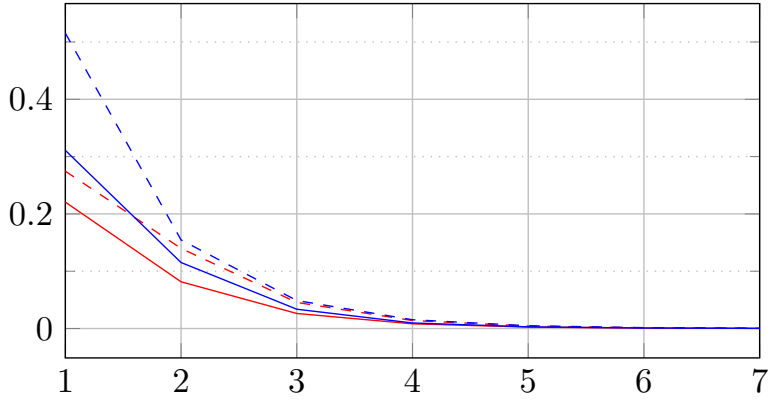

Fig. 3. Linear scale stopping criteria evolution for Algorithm 1 ( $\longrightarrow$ ) and Algorithm 2 (-). Solid lines correspond to $\left\|R_{i}\left(I-X_{i} Y_{i}\right)\right\|$ and dashed lines to $\left\|\left(I-X_{i} Y_{i-1}\right) Q_{i}\right\|$.

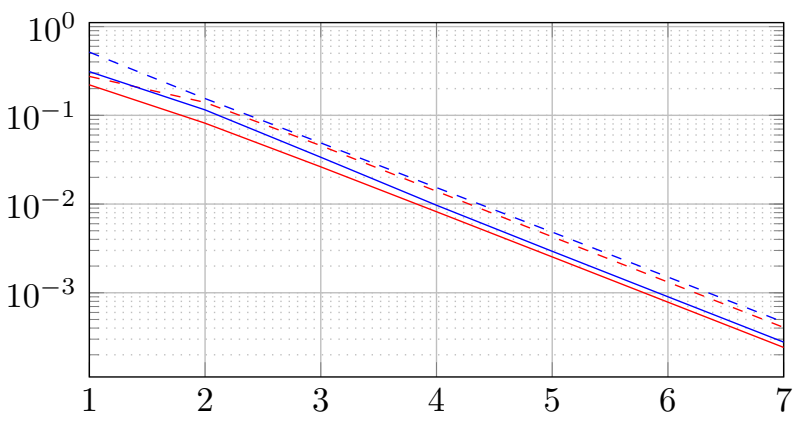

Fig. 4. Logarithmic scale stopping criteria evolution for Algorithm 1 ( - ) and Algorithm 2 (- $)$. Solid lines correspond to $\| R_{i}\left(I-X_{i} Y_{i}\right) \mid$ and dashed lines to $\left\|\left(I-X_{i} Y_{i-1}\right) Q_{i}\right\|$.

respectively to aid analysis. A normalization error metric is also provided in Table I to quantify how close to a true normalized BCF the obtained solution is. For the pair $\{L, M\}$, this error is defined as $\left\|I-[M-L][M-L]^{\sim}\right\|_{\infty}$; this is similarly defined for the pair $\{N, M\}$. Figure 5 shows the evolution of $\rho\left(X_{i} X_{i-1}^{-1}\right)$ and $\rho\left(Y_{i} Y_{i-1}^{-1}\right)$ for this example. It can be seen that, like in the previous example, the sequence of generated Gramians is still not monotonic. Both algorithms converged to the same solution (within tolerance) in the same number of iterations.

Though it seems from Table I and Figures 3 and 4 that the two algorithms perform very similarly in a discrete time context, the real-time performance of Algorithm 2 was found to be significantly better terminating in within $3.1 \mathrm{~ms}$ while Algorithm 1 required approximately $11.3 \mathrm{~ms}$.

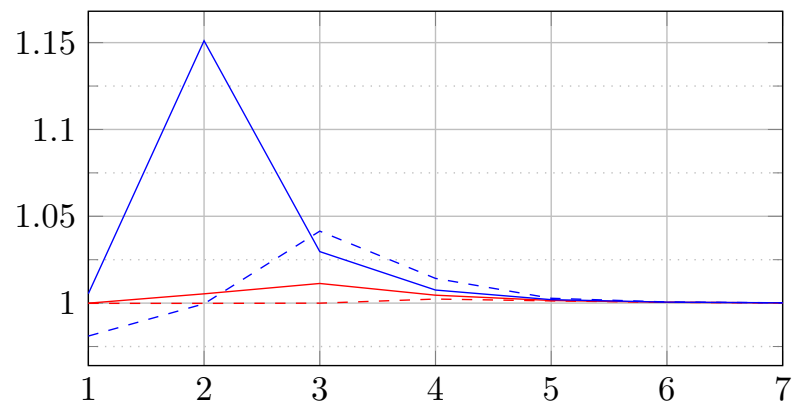

Fig. 5. Evolution of $\rho\left(X_{i} X_{i-1}^{-1}\right)$ (dashed) and $\rho\left(Y_{i} Y_{i-1}^{-1}\right)$ (solid) for Algorithm 1 ( $\longrightarrow$ ) and Algorithm 2 ( - ). 


\begin{tabular}{cc}
\hline \multicolumn{1}{c}{ Parameter } & Value \\
\hline$\left\|X_{R}-X_{K}\right\|$ & $1.320 \times 10^{-5}$ \\
$\left\|Y_{R}-Y_{K}\right\|$ & $1.334 \times 10^{-5}$ \\
$\left\|Q_{R}-Q_{K}\right\|$ & $2.664 \times 10^{-5}$ \\
$\left\|R_{R}-R_{K}\right\|$ & $1.592 \times 10^{-5}$ \\
\hline
\end{tabular}

TABLE II

SOLUTION DIFFERENCE. THE SUBSCRIPTS $R$ AND $K$ DENOTE MATRICES OBTAINED FROM ALGORITHMS 1 AND 2 RESPECTIVELY.

Table II lists the norm difference in the parameters obtained using each of the two algorithms. As stated above and and exemplified by this table, both algorithms converged to the same normalizing pair $Q$ and $R$. The resulting pair was given by

$$
\begin{gathered}
Q=\left[\begin{array}{llll}
0.390 & 0.273 & 0.398 & 0.281
\end{array}\right]^{*} \text { and } \\
R=\left[\begin{array}{llll}
-0.572 & -0.383 & -0.487 & -0.273
\end{array}\right],
\end{gathered}
$$

with $Q^{*} C^{*} C Q=0.2266$ and $R B B^{*} R^{*}=0.2264$. The resulting associated Gramians were given by

$$
\begin{aligned}
X & =\left[\begin{array}{llll}
0.432 & 0.232 & 0.076 & 0.062 \\
0.232 & 0.284 & 0.044 & 0.037 \\
0.076 & 0.044 & 0.618 & 0.134 \\
0.062 & 0.037 & 0.134 & 0.610
\end{array}\right]>0 \text { and } \\
Y & =\left[\begin{array}{llll}
1.048 & 0.171 & 0.248 & 0.063 \\
0.171 & 0.742 & 0.147 & 0.195 \\
0.248 & 0.147 & 0.675 & 0.290 \\
0.063 & 0.195 & 0.290 & 0.284
\end{array}\right]>0 .
\end{aligned}
$$

With the pair normalizing $Q$ and $R$ generated above, the eigenvalues of $A+Q R$ are placed at $-0.4519 \pm 2.0078 i$ and $-0.4072 \pm 0.9122 i$. The induced $Q R$-BCF was then constructed as

$$
\begin{aligned}
& {\left[\begin{array}{c:c}
M & -L \\
\hdashline N & K
\end{array}\right]=} \\
& {\left[\begin{array}{cccc|c:cc}
-0.223 & -0.149 & 0.810 & -0.106 & 0.390 & 0 & 0 \\
-0.156 & -0.104 & -0.133 & 0.926 & 0.273 & 0 & 0 \\
-2.028 & 1.248 & -0.514 & 0.131 & 0.398 & 0.8 & 0 \\
1.589 & -3.358 & 0.163 & -0.877 & 0.281 & 0 & 1 \\
\hdashline-0.572 & -0.383 & -0.487 & -0.273 & 1 & 0 & 0 \\
\hdashline 1 & 0 & 0 & 0 & 0 & 0 & 0 \\
0 & 1 & 0 & 0 & 0 & 0 & 0
\end{array}\right]}
\end{aligned}
$$

\section{CONCLUSION}

In this paper, the concept of normalized bicoprime factorizations is introduced. Conditions are presented for a factorization obtained via the $Q R$-BCF parametrization to be normalized. These conditions are then used as the basis for two iterative algorithms that can be used to construct a normalized BCF of a plant.

Although the concept of normalized BCFs is thoroughly examined, the motivation behind this study might be unclear to the reader. One possible application of such factorizations is that of robust control synthesis. In fact, it can be shown that given a normalized $\mathrm{BCF}$ of the plant, a lower bound on the achievable robust stability margin with respect to $\mathrm{BCF}$ uncertainty as defined by [9] is given by

$$
\max \left\{\left(1-\left\|\left[\begin{array}{c}
M \\
N
\end{array}\right]\right\|_{H}^{2}\right)^{-\frac{1}{2}},\left(1-\left\|\left[\begin{array}{ll}
M & -L
\end{array}\right]\right\|_{H}^{2}\right)^{-\frac{1}{2}}\right\}
$$

where $\|S\|_{H}$ corresponds to the Hankel norm of the transfer matrix $S \in \mathscr{R} \mathscr{H}_{\infty}$. The above fact will not be proven here as it is out of the scope of this paper.

Another possible usage of normalized BCF is in the context of distance measures as defined by [4], [18]. Using the standard procedures presented therein it is not possible to capture the notion of distance between two plants based on their BCFs. It is possible that imposing a normalization property is the tool needed to achieve this.

\section{REFERENCES}

[1] K. Glover and D. McFarlane, "Robust stabilization of normalized coprime factor plant descriptions with $\mathscr{H}_{\infty}$-bounded uncertainty," IEEE Transactions on Automatic Control, vol. 34, no. 8, pp. 821-830, Aug. 1989.

[2] D. C. McFarlane and K. Glover, Robust Controller Design Using Normalized Coprime Factor Plant Descriptions. Berlin: SpringerVerlag, 1990.

[3] D. McFarlane and G. K., "A loop shaping design procedure using $H_{\infty}$ synthesis," IEEE Transactions on Automatic Control, vol. 37, no. 6 , pp. 759-769, Jun. 1992.

[4] A. Lanzon and G. Papageorgiou, "Distance measures for uncertain linear systems: A general theory," IEEE Transactions on Automatic Control, vol. 54, no. 7, pp. 1532-1547, Jul. 2009.

[5] G. Vinnicombe, Uncertainty and Feedback. $\mathscr{H}_{\infty}$ loopshaping and the $\nu$-gap metric. London: Imperial College Press, 2001.

[6] K. Zhou, J. C. Doyle, and K. Glover, Robust and Optimal Control. Upper Saddle River, New Jersey: Prentice-Hall, Inc., 1996.

[7] M. Vidyasagar, Control System Synthesis: A Factorization Approach. Morgan \& Claypool Publishers, 2011, reprint.

[8] M. Tsiakkas and A. Lanzon, "Bicoprime factor stability criteria and uncertainty characterisation," in Proceedings of the 8th IFAC Symposium on Robust Control Design, Bratislava, Slovakia, Jul. 2015, pp. 228-233.

[9] _ _ "Foundations of a bicoprime factorization theory," IEEE Transactions on Automatic Control, vol. 62, no. 9, pp. 4598-4603, Sept 2017.

[10] C. N. Nett, C. A. Jacobson, and M. J. Balas, "A connection between state-space and doubly coprime fractional representations," IEEE Transactions on Automatic Control, vol. 29, pp. 831-832, 1984.

[11] D. Meyer and G. Franklin, "A connection between normalized coprime factorizations and linear quadratic regulator theory," IEEE Transactions on Automatic Control, vol. 32, no. 3, pp. 227-228, Mar 1987.

[12] M. Vidyasagar, "Normalized coprime factorizations for nonstrictly proper systems," IEEE Transactions on Automatic Control, vol. 33, no. 3, pp. 300-301, Mar. 1988.

[13] D. Kleinman, "On an iterative technique for riccati equation computations," IEEE Transactions on Automatic Control, vol. 13, no. 1, pp. 114-115, Feb 1968.

[14] A. J. Laub, Matrix Analysis for Scientists \& Engineers. Society for Industrial and Applied Mathematics, 2005.

[15] H. T. Banks and K. Ito, "A numerical algorithm for optimal feedback gains in high dimensional linear quadratic regulator problems," SIAM Journal on Control and Optimization, vol. 29, no. 3, pp. 499-515, 1991.

[16] A. Lanzon, Y. Feng, B. D. O. Anderson, and M. Rotkowitz, "Computing the positive stabilizing solution to Algebraic Riccati Equations with an indefinite quadratic term via a recursive method," IEEE Transactions on Automatic Control, vol. 53, no. 10, pp. 2280-2291, Nov. 2008.

[17] R. Horn and C. Johnson, Matrix Analysis, 2nd ed. Cambridge University Press, 2012.

[18] A. Lanzon, S. Engelken, S. Patra, and G. Papageorgiou, "Robust stability and performance analysis for uncertain linear systems - The distance measure approach," International Journal of Robust and Nonlinear Control, vol. 22, no. 11, pp. 1270-1292, Jul. 2012. 